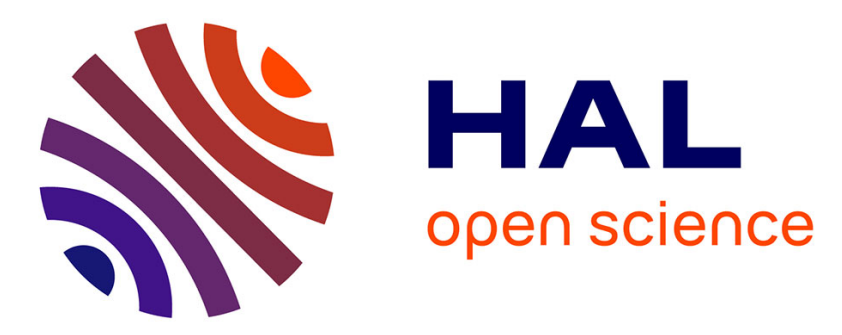

\title{
Derivation of an Analytical Expression of the Gaussian Model Statistical Resolution Limit
}

\author{
Messaoud Thamery, Remy Boyer, Karim Abed-Meraim
}

\section{To cite this version:}

Messaoud Thamery, Remy Boyer, Karim Abed-Meraim. Derivation of an Analytical Expression of the Gaussian Model Statistical Resolution Limit. ICASSP 2013 - 38th IEEE International Conference on Acoustics, Speech and Signal Processing, IEEE, Apr 2013, Vancouver, Canada. 10.1109/icassp.2013.6638721 . hal-01002335

\section{HAL Id: hal-01002335 \\ https://hal.science/hal-01002335}

Submitted on 13 Jan 2015

HAL is a multi-disciplinary open access archive for the deposit and dissemination of scientific research documents, whether they are published or not. The documents may come from teaching and research institutions in France or abroad, or from public or private research centers.
L'archive ouverte pluridisciplinaire HAL, est destinée au dépôt et à la diffusion de documents scientifiques de niveau recherche, publiés ou non, émanant des établissements d'enseignement et de recherche français ou étrangers, des laboratoires publics ou privés. 


\section{DERIVATION OF AN ANALYTICAL EXPRESSION OF THE GAUSSIAN MODEL STATISTICAL RESOLUTION LIMIT}

\author{
Messaoud Thameri \\ TELECOM ParisTech \\ TSI Department \\ Paris, France
}

\author{
Rémy Boyer \\ Université Paris Sud XI \\ LSS/SUPELEC \\ Gif Sur Yvette, France
}

\author{
Karim Abed-Meraim \\ Polytech'Orléans \\ PRISME Laboratory \\ Orléans, France.
}

\begin{abstract}
Statistical Resolution Limit (SRL), defined as the minimal separation to resolve two closely spaced signals, is one of the important tools to evaluate a given system performance. Based on S.T. Smith's formulation of the SRL, this paper provides a methodology to compute an approximate analytical expression of the resolution limit in the Gaussian model case. As an application, we consider the particular case of two sources located in the near field and consider the resolution limit in terms of minimum angular separation. Discussion and numerical illustrations are then given to get more insights on the proposed derivation and to validate our theoretical results.
\end{abstract}

Index Terms- CRB, Gaussian model, SRL, NFL

\section{INTRODUCTION}

The SRL is a measure of the minimal difference needed for two closely spaced parameters (sources) to be detected and consequently correctly estimated and separated [1]. The SRL, as defined in [2], is related to a given statistical parametric model for the observed signal and hence it represents an inherent lower bound for the resolution independently of the considered estimation method.

Many authors have considered the SRL for different applications including MIMO radar [3], spectral estimation [4], DOA (Direction Of Arrival) estimation [5, 6], near field source localization [7] and for passive Polarized Source Localization [8]. Different approaches have been used for the derivation of the SRL. In [2], the evaluation of the SRL $\delta$ is based on the estimation accuracy and is obtained by solving the equation $\delta=\sqrt{\mathrm{CRB}(\delta)}$ where CRB refers to the model's Cramer Rao Bound. The second approach considered in $[9,10]$ is based on the detection theory. Interestingly, the two approaches are shown in [6] to be closely related and can be unified by defining the SRL as the solution of the following equation

$$
\delta=\gamma \sqrt{\operatorname{CRB}(\delta)}
$$

where $\gamma$ is a proportionality constant corresponding to a given couple of detection and false alarm probabilities.
To our best knowledge, this paper is the first to propose analytical expressions for conditional and unconditional Gaussian models. More precisely, in this paper, we derive analytical expressions for the SRL (i.e. solution of equation (1)) based on the Taylor expansion of the CRB. The proposed SRL expression can be used under certain system conditions that are commented in this paper. Alternatively, when the analytical SRL formula cannot be used, we proposed to use a numerical solution of (1) based on the fixed point method [11]. Both numerical and analytical solutions are compared and discussed through numerical simulations.

\section{PROBLEM FORMULATION}

We consider i.i.d. multivariate observation vectors $\mathbf{x}(t), t=$ $0, \cdots, T-1$ with a complex circular Gaussian probability density function of covariance $\mathbf{R}(\boldsymbol{\theta})$ and mean value $\boldsymbol{\mu}(\boldsymbol{\theta})$ where $\boldsymbol{\theta}$ is the global parameter vector that can be written as:

$$
\boldsymbol{\theta}^{T}=\left[\boldsymbol{\theta}_{1}^{T}, \boldsymbol{\theta}_{2}^{T}, \cdots, \boldsymbol{\theta}_{q}^{T}, \boldsymbol{\theta}_{n}^{T}\right]
$$

$\boldsymbol{\theta}_{i}, i=1, \cdots, q$ corresponds to the unknown parameter vector of the $i^{t h}$ signal component (e.g. a given source in the array processing context or a sinusoidal component in a spectral analysis context) and $\boldsymbol{\theta}_{n}$ represents the nuisance (non desired) parameter vector. In array processing, such a signal is written as $\mathbf{x}(t)=\mathbf{A}(\boldsymbol{\theta}) \mathbf{s}(t)+\mathbf{n}(t)$ and can represent:

1. either the conditional model in which the source signals $\mathbf{s}(t)$ are assumed to be deterministic and the noise $\mathbf{n}(t)$ is Gaussian distributed. The desired parameter vector $\boldsymbol{\theta}_{i}, i=1, \cdots, q$ represents the source location parameters (e.g. angles of arrival, range, Doppler, etc) and its related statistical information is given by the mean value $\boldsymbol{\mu}(\boldsymbol{\theta})$,

2. or the unconditional model in which we assume that the source signals are complex circular Gaussian with zero mean and unknown covariance $\mathbf{R}_{s}$. In that case, we have $\boldsymbol{\mu}(\boldsymbol{\theta})=\mathbf{0}$ and all statistical information is contained in the covariance matrix $\mathbf{R}(\boldsymbol{\theta})$. 
Our main objective is to express analytically the SRL of. one of the source parameters by using a Taylor expansion of (1) as explained next.

\section{DERIVATION OF AN ANALYTICAL SRL}

\subsection{Review of the SRL problem}

In [2], Smith proposed the following SRL definition: Two signals are resolvable w.r.t. the DOA if the difference between the DOA is greater than the standard deviation of the DOA difference estimation according to the $C R B$. This criterion has been extended in [6] within a generalized framework unifying it with the hypothesis testing-based approaches and leading to the criterion in (1). In [12, 13], the multiple parameter case is considered and the SRL formulation has been generalized and expressed as the minimum distance between two source parameter vectors for the signal components (sources) to be separable.

In all cases, the SRL is formulated as a solution of a non linear equation and solved numerically using standard optimization methods. Explicit SRL expressions have been given only in very specific, simplified contexts as the one considered in [8] where source orthogonality is assumed as a simplifying hypothesis.

Now, Numerical solutions might be too expensive in high dimensional cases due to the FIM (Fisher Information Matrix) inversion involved in the computation of the CRB. Next, we propose to solve equation (1) based on a second order Taylor expansion of the CRB which leads to approximate analytical expressions of the SRL. In the sequel, we re-parametrize our model as

$$
\boldsymbol{\kappa}=f(\boldsymbol{\theta})=\left[\begin{array}{ll}
\delta & \tilde{\boldsymbol{\theta}}^{T}
\end{array}\right]^{T}
$$

where we set, without loss of generality, $\delta=\boldsymbol{\theta}_{1}(1)-\boldsymbol{\theta}_{2}(1)$ and $\tilde{\boldsymbol{\theta}}$ is the vector of all remaining parameters independent of $\delta$.

\subsection{Taylor expansion of the FIM}

Before computing the second order Taylor expansion of the CRB, we compute first the second order Taylor expansion of the FIM. For the Gaussian model, the $(l, m)^{t h}$ entry of the FIM (denoted $\mathbf{F}$ ) with respect to the parameter vector $\kappa$ is given by [14]

$[\mathbf{F}(\boldsymbol{\kappa})]_{l m}=\operatorname{Ttr}\left\{\mathbf{R}^{-1} \frac{\partial \mathbf{R}}{\partial \kappa_{l}} \mathbf{R}^{-1} \frac{\partial \mathbf{R}}{\partial \kappa_{m}}\right\}+2 \Re\left\{\frac{\partial \boldsymbol{\mu}^{H}}{\partial \kappa_{l}} \mathbf{R}^{-1} \frac{\partial \boldsymbol{\mu}}{\partial \kappa_{m}}\right\}$

where $\operatorname{tr}($.$) is the trace operator and \Re($.$) refers to the real$ part of a complex entity. Since the distribution of the observed data is expressed as a function of $\delta$, hence the FIM can also be expressed as a function of $\delta$ and one can easily compute its second order Taylor expansion as

$$
\mathbf{F}=\mathbf{F}_{0}+\mathbf{F}_{1} \delta+\frac{1}{2} \mathbf{F}_{2} \delta^{2}+O\left(\delta^{3}\right)
$$

where $O()$ summarizes the least-significant terms in Taylor expansion when $\delta$ is close enough to 0 and the specific computation of $\mathbf{F}_{0}=\mathbf{F}(\delta=0), \mathbf{F}_{1}=\left.\frac{\partial \mathbf{F}}{\partial \delta}\right|_{\delta=0}$ and $\mathbf{F}_{2}=$ $\left.\frac{\partial^{2} \mathbf{F}}{\partial \delta^{2}}\right|_{\delta=0}$ depends on the case at hand.

For the unconditional model, we have :

$$
\begin{aligned}
{\left[\mathbf{F}_{0}\right]_{i j} } & =\operatorname{Ttr}\left\{\mathbf{R}^{-1} \frac{\partial \mathbf{R}}{\partial \kappa_{i}} \mathbf{R}^{-1} \frac{\partial \mathbf{R}}{\partial \kappa_{j}}\right\}_{\delta=0} \\
{\left[\mathbf{F}_{1}\right]_{i j} } & =\frac{\partial}{\partial \delta}\left[\operatorname{Ttr}\left\{\mathbf{R}^{-1} \frac{\partial \mathbf{R}}{\partial \kappa_{i}} \mathbf{R}^{-1} \frac{\partial \mathbf{R}}{\partial \kappa_{j}}\right\}\right]_{\delta=0} \\
& =\mathbf{F}_{p 1}+\mathbf{F}_{p 1}^{T}+\mathbf{F}_{p 2}+\mathbf{F}_{p 2}^{T} \\
{\left[\mathbf{F}_{2}\right]_{i j} } & =\frac{\partial^{2}}{\partial \delta^{2}}\left[\operatorname{Ttr}\left\{\mathbf{R}^{-1} \frac{\partial \mathbf{R}}{\partial \kappa_{i}} \mathbf{R}^{-1} \frac{\partial \mathbf{R}}{\partial \kappa_{j}}\right\}\right]_{\delta=0} \\
& =\mathbf{F}_{s 1}+\mathbf{F}_{s 1}^{T}+2\left(\mathbf{F}_{s 2}+\mathbf{F}_{s 2}^{T}\right)+2 \mathbf{F}_{s 3}+2\left(\mathbf{F}_{s 4}+\mathbf{F}_{s 4}^{T}\right) \\
& +2 \mathbf{F}_{s 5}+\mathbf{F}_{s 6}+\mathbf{F}_{s 6}^{T}
\end{aligned}
$$

where

$$
\begin{aligned}
& {\left[\mathbf{F}_{p 1}\right]_{l m}=\operatorname{Ttr}\left\{-\mathbf{R}^{-1} \frac{\partial \mathbf{R}}{\partial \delta} \mathbf{R}^{-1} \frac{\partial \mathbf{R}}{\partial \kappa_{l}} \mathbf{R}^{-1} \frac{\partial \mathbf{R}}{\partial \kappa_{m}}\right\}_{\delta=0}} \\
& {\left[\mathbf{F}_{p 2}\right]_{l m}=\operatorname{Ttr}\left\{\mathbf{R}^{-1} \frac{\partial^{2} \mathbf{R}}{\partial \delta \partial \kappa_{l}} \mathbf{R}^{-1} \frac{\partial \mathbf{R}}{\partial \kappa_{m}}\right\}_{\delta=0}} \\
& {\left[\mathbf{F}_{s 1}\right]_{l m}=\operatorname{Ttr}\left\{\left(-\mathbf{R}^{-1} \frac{\partial^{2} \mathbf{R}}{\partial \delta^{2}} \mathbf{R}^{-1}+2 \mathbf{R}^{-1} \frac{\partial \mathbf{R}}{\partial \delta} \mathbf{R}^{-1} \frac{\partial \mathbf{R}}{\partial \delta} \mathbf{R}^{-1}\right) \frac{\partial \mathbf{R}}{\partial \kappa_{l}} \mathbf{R}^{-1} \frac{\partial \mathbf{R}}{\partial \kappa_{m}}\right\}_{\delta=0}} \\
& {\left[\mathbf{F}_{s 2}\right]_{l m}=\operatorname{Ttr}\left\{-\mathbf{R}^{-1} \frac{\partial \mathbf{R}}{\partial \delta} \mathbf{R}^{-1} \frac{\partial^{2} \mathbf{R}}{\partial \delta \partial \kappa_{l}} \mathbf{R}^{-1} \frac{\partial \mathbf{R}}{\partial \kappa_{m}}\right\}_{\delta=0}} \\
& {\left[\mathbf{F}_{s 3}\right]_{l m}=\operatorname{Ttr}\left\{\mathbf{R}^{-1} \frac{\partial \mathbf{R}}{\partial \delta} \mathbf{R}^{-1} \frac{\partial \mathbf{R}}{\partial \kappa_{l}} \mathbf{R}^{-1} \frac{\partial \mathbf{R}}{\partial \delta} \mathbf{R}^{-1} \frac{\partial \mathbf{R}}{\partial \kappa_{m}}\right\}_{\delta=0}} \\
& {\left[\mathbf{F}_{s 4}\right]_{l m}=\operatorname{Ttr}\left\{-\mathbf{R}^{-1} \frac{\partial \mathbf{R}}{\partial \delta} \mathbf{R}^{-1} \frac{\partial \mathbf{R}}{\partial \kappa_{l}} \mathbf{R}^{-1} \frac{\partial^{2} \mathbf{R}}{\partial \delta \partial \kappa_{m}}\right\}_{\delta=0}} \\
& {\left[\mathbf{F}_{s 5}\right]_{l m}=\operatorname{Ttr}\left\{\mathbf{R}^{-1} \frac{\partial^{2} \mathbf{R}}{\partial \delta \partial \kappa_{l}} \mathbf{R}^{-1} \frac{\partial^{2} \mathbf{R}}{\partial \delta \partial \kappa_{m}}\right\}_{\delta=0}} \\
& {\left[\mathbf{F}_{s 6}\right]_{l m}=\operatorname{Ttr}\left\{\mathbf{R}^{-1} \frac{\partial^{2}}{\partial \delta^{2}}\left(\frac{\partial \mathbf{R}}{\partial \kappa_{l}}\right) \mathbf{R}^{-1} \frac{\partial \mathbf{R}}{\partial \kappa_{m}}\right\}_{\delta=0}}
\end{aligned}
$$

For the conditional model with a white Gaussian noise of covariance matrix $\sigma^{2} \mathbf{I}$, we have:

$$
\begin{aligned}
{\left[\mathbf{F}_{0}\right]_{l m} } & =\frac{N T}{\sigma^{4}}\left\{\frac{\partial \sigma^{2}}{\partial \kappa_{l}} \frac{\partial \sigma^{2}}{\partial \kappa_{m}}\right\}_{\delta=0}+\frac{2}{\sigma^{2}} \Re\left\{\frac{\partial \boldsymbol{\mu}^{H}}{\partial \kappa_{l}} \frac{\partial \boldsymbol{\mu}}{\partial \kappa_{m}}\right\}_{\delta=0} \\
{\left[\mathbf{F}_{1}\right]_{l m} } & =\frac{2}{\sigma^{2}}\left\{\frac{\partial^{2} \boldsymbol{\mu}^{H}}{\partial \delta \partial \kappa_{l}} \frac{\partial \boldsymbol{\mu}}{\partial \kappa_{m}}\right\}_{\delta=0}+\frac{2}{\sigma^{2}} \Re\left\{\frac{\partial \boldsymbol{\mu}^{H}}{\partial \kappa_{l}} \frac{\partial^{2} \boldsymbol{\mu}}{\partial \delta \partial \kappa_{m}}\right\}_{\delta=0} \\
{\left[\mathbf{F}_{2}\right]_{l m} } & =\frac{2}{\sigma^{2}} \Re\left\{\frac{\partial^{3} \boldsymbol{\mu}^{H}}{\partial \delta^{3} \partial \kappa_{l}} \frac{\partial \boldsymbol{\mu}}{\partial \kappa_{m}}\right\}_{\delta=0}+\frac{4}{\sigma^{2}} \Re\left\{\frac{\partial^{2} \boldsymbol{\mu}^{H}}{\partial \delta \partial \kappa_{l}} \frac{\partial^{2} \boldsymbol{\mu}}{\partial \delta \partial \kappa_{m}}\right\}_{\delta=0} \\
& +\frac{2}{\sigma^{2}} \Re\left\{\frac{\partial \boldsymbol{\mu}^{H}}{\partial \kappa_{l}} \frac{\partial^{3} \boldsymbol{\mu}}{\partial \delta^{3} \partial \kappa_{m}}\right\}_{\delta=0}
\end{aligned}
$$

\subsection{Taylor expansion of the CRB}

The CRB is computed as the inverse of the FIM and its second order Taylor expansion can be achieved according to the following lemma [15]

Lemma 3.1. If $\mathbf{M}$ is asymptotically nilpotent, i.e. $\mathbf{M}^{n} \rightarrow \mathbf{0}$ when $n \rightarrow \infty$, then the following Taylor expansion holds:

$$
(\mathbf{I}+\mathbf{M})^{-1}=\mathbf{I}-\mathbf{M}+\mathbf{M}^{2}-\mathbf{M}^{3}+\cdots
$$


Now, under the assumption that $\mathbf{F}_{0}$ is non singular, this lemma can be used for the Taylor expansion of the CRB (denoted $\mathbf{C}$ ) according to

$$
\begin{aligned}
\mathbf{C} & =\mathbf{F}^{-1}=\left(\mathbf{I}+\mathbf{F}_{0}^{-1} \mathbf{B}\right)^{-1} \mathbf{F}_{0}^{-1} \\
& =\left(\mathbf{I}-\mathbf{F}_{0}^{-1} \mathbf{B}+\left(\mathbf{F}_{0}^{-1} \mathbf{B}\right)^{2}\right) \mathbf{F}_{0}^{-1}+O\left(\delta^{3}\right)
\end{aligned}
$$

where $\mathbf{B}=\mathbf{F}_{1} \delta+\frac{1}{2} \mathbf{F}_{2} \delta^{2}$. Replacing $\mathbf{B}$ by its expression in (7) leads to

$$
\begin{aligned}
\mathbf{C} & =\mathbf{C}_{0}+\mathbf{C}_{1} \delta+\mathbf{C}_{2} \delta^{2}+O\left(\delta^{3}\right) \\
\mathbf{C}_{0} & =\mathbf{F}_{0}^{-1} \\
\mathbf{C}_{1} & =-\mathbf{F}_{0}^{-1} \mathbf{F}_{1} \mathbf{F}_{0}^{-1} \\
\mathbf{C}_{2} & =\mathbf{F}_{0}^{-1} \mathbf{F}_{1} \mathbf{F}_{0}^{-1} \mathbf{F}_{1} \mathbf{F}_{0}^{-1}-\frac{1}{2} \mathbf{F}_{0}^{-1} \mathbf{F}_{2} \mathbf{F}_{0}^{-1}
\end{aligned}
$$

This result is verified under the following hypotheses:

(H1): $\quad \mathbf{F}_{0}$ is non singular.

(H2) : $\quad \mathbf{F}_{0}^{-1} \mathbf{B}$ is asymptotically nilpotent.

Hypothesis (H1) is generally verified if at least one of their parameters of interest of the considered sources are different, i.e. if $\boldsymbol{\theta}_{1} \neq \boldsymbol{\theta}_{2}$. For example, in the near field localization (NFL) problem considered in section 4 , the sources are 'separable' as long as their location is different, i.e. they have either different angles or different ranges. Hypothesis (H1) is also verified in the case where extra source information is available, which is the case for example if one of the source location is a priori known [6]. In the case where $\mathbf{F}_{0}$ is singular, one can always use a kind of 'regularization' by writing $\mathbf{F}=\left(\mathbf{F}_{0}+\epsilon \mathbf{I}\right)-\epsilon \mathbf{I}+\mathbf{F}_{1} \delta+\frac{1}{2} \mathbf{F}_{2} \delta^{2}+O\left(\delta^{3}\right)$ where $\epsilon<<1$ is a chosen regularization parameter, in such a way we invert $\left(\mathbf{F}_{0}+\epsilon \mathbf{I}\right)$ instead of $\mathbf{F}_{0}$ in the Taylor expansion given in equation (7). For the second hypothesis (H2) to hold, one needs often to assume that $\delta<<1$, this is true in many situations but not in the adverse ones (small array size, small sample size, very low SNRs, etc) as shown by our simulation results.

\subsection{Approximate analytical SRL expression}

Now, replacing the CRB by its second order Taylor expansion in the equation $\delta^{2}=\gamma^{2} \mathrm{CRB}(\delta)$, leads to a simple polynomial rooting problem according to

$$
\left[\mathbf{C}_{0}\right]_{11}+\left[\mathbf{C}_{1}\right]_{11} \delta+\left(\left[\mathbf{C}_{2}\right]_{11}-\frac{1}{\gamma^{2}}\right) \delta^{2}=0
$$

where $[\mathbf{C}]_{i j}$ refers to the $(i, j)^{t h}$ entry of matrix $\mathbf{C}$. The roots of (9) are given by

$$
\delta_{ \pm}=\frac{-\left[\mathbf{C}_{1}\right]_{11} \pm \sqrt{\Delta}}{2\left(\left[\mathbf{C}_{2}\right]_{11}-\frac{1}{\gamma^{2}}\right)}
$$

with $\Delta=\left[\mathbf{C}_{1}\right]_{11}^{2}-4\left(\left[\mathbf{C}_{2}\right]_{11}-\frac{1}{\gamma^{2}}\right)\left[\mathbf{C}_{0}\right]_{11}$.
The SRL is computed as the absolute value of the solution of (9) with the smallest absolute value which is

$$
S R L=\left|\frac{-\left[\mathbf{C}_{1}\right]_{11}+\operatorname{sign}\left(\left[\mathbf{C}_{1}\right]_{11}\right) \sqrt{\Delta}}{2\left(\left[\mathbf{C}_{2}\right]_{11}-\frac{1}{\gamma^{2}}\right)}\right|
$$

\subsection{Discussion}

Below, we provide some comments to clarify certain aspects of the proposed SRL derivation approach.

- As shown earlier, equation (9) has generally two real valued solutions and we selected the one with the smallest absolute value. Indeed, since the Taylor expansion is valid only in a small interval centered at zero, one needs to select the meaningful solution within this interval and disregard any other polynomial root which does not correspond to our approximate equation. This choice corresponds well with the observations made in our simulation experiments.

- The analytical SRL solution in (9) should be validated only if $\Delta \geq 0$ (since $\delta$ is real valued) and if hypothesis (H2) is met, i.e. one should check a posteriori that matrix $\mathbf{F}_{0}^{-1} \mathbf{B}$ is asymptotically nilpotent. In the case where $\Delta<0$ or if condition (H2) is not met one should disregard the solution of (9) and consider a numerical method to solve (1).

- The usefulness of the analytical SRL resides in its potential use for interpretable expressions ${ }^{1}$ and for reducing the numerical cost when the FIM is of large dimensional matrix form. For example, in the deterministic NFL problem with 2 sources and $T=100$ samples, we will have an FIM of size $404 \times 404$. Fixed point method needs several iterations to converge, each of them requires the inversion of this large FIM. By cons, our approach needs only one inversion of the FIM.

\section{NUMERICAL RESULTS}

In this section, we illustrate our approach by considering a particular case where a uniform linear array (ULA) with $N$ sensors receives the signals emitted by two narrow band sources located in the near field. The observed signal is corrupted by additive complex circular white Gaussian noise of zero mean and covariance matrix $\sigma^{2} \mathbf{I}_{N}$. Hence, the observed vector is expressed by [16]

$$
\mathbf{x}(t)=\mathbf{A} \mathbf{s}(t)+\mathbf{n}(t)
$$

where $\mathbf{s}(t)=\left[s_{1}(t) s_{2}(t)\right]^{T}, s_{i}(t)$ is assumed to be Gaussian with zero mean and variance $\sigma_{s_{i}}^{2}, \mathbf{A}=\left[\begin{array}{ll}\mathbf{a}_{1} & \mathbf{a}_{2}\end{array}\right], \mathbf{a}_{i}=$

\footnotetext{
${ }^{1}$ This is the focus of our future work, where some interesting developments of the SRL expression in the NFL case will be provided.
} 


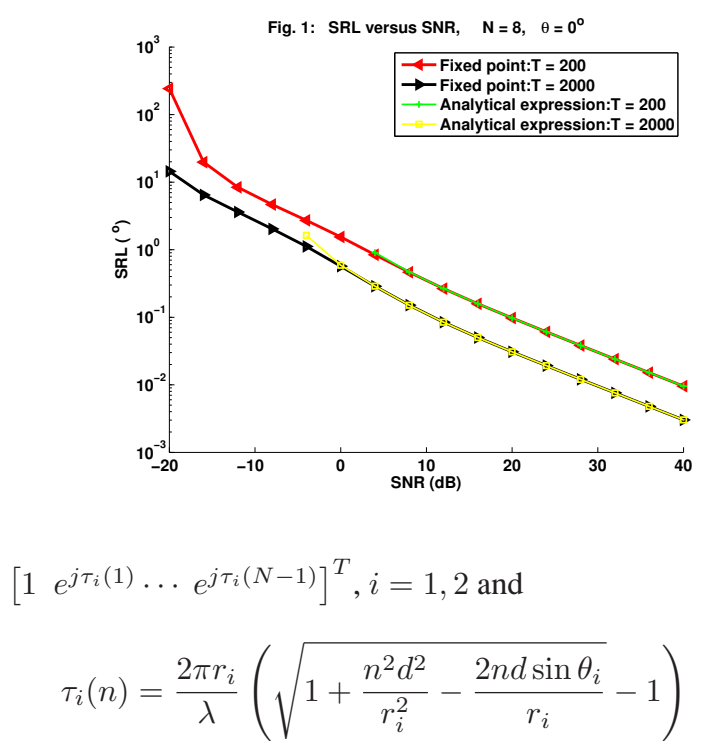

where $d$ is the inter element spacing, $\lambda$ is the propagation wavelength and $\left(r_{i}, \theta_{i}\right), i=1,2$, are the polar coordinates of the sources. In the following, we are interested only by the Angular Resolution Limit (ARL). The numerical SRL is computed using fixed point method, and is presented by red and black curves in both Fig. 1 and Fig. 2 and by red curve in the remaining figures.

Fig. 1 and Fig. 2 present the variation of the SRL w.r.t. the SNR for different values of $N$ and $T$. One can observe that: $(i)$ the analytical SRL fits well with the numerical one in the non-adverse situations and for a large range of system parameter values; $(i i)$ in the adverse situations (very low SNRs, small sample size or small antenna size) the fitting between the analytical and numerical SRL expressions is lost. In that case, we observe that for the analytical SRL, hypothesis (H2) is not satisfied or the discriminant $\Delta$ is negative valued, this corresponds to the intervals where the analytical SRL plots are truncated. In the adverse cases, we have used the fixed point method to compute the solution of (1). We have observed a very fast convergence of the latter method (typically, we have less than 10 iterations in our simulation context. Finally, (iii) we can see from this experiment that the range of 'validity' of the analytical SRL expression increases when the context becomes more favorable: For example, for $T=200$ and $N=8$, we observe that the SRL expression is valid if the SNR is higher than $2 \mathrm{~dB}$ while for $T=200$ and $N=20$ it is valid for an SNR approximately higher than $-16 \mathrm{~dB}$.

Fig. 3, 4 and 5 illustrate the variation of the SRL w.r.t. different system parameters, namely the look up direction, the number of sensors and the sample size, respectively. One can observe, in particular, that the SRL increases significantly in the lateral directions (i.e. when $|\theta| \rightarrow 90^{\circ}$ ) as compared to the central direction (i.e. when $|\theta| \rightarrow 0^{\circ}$ ).
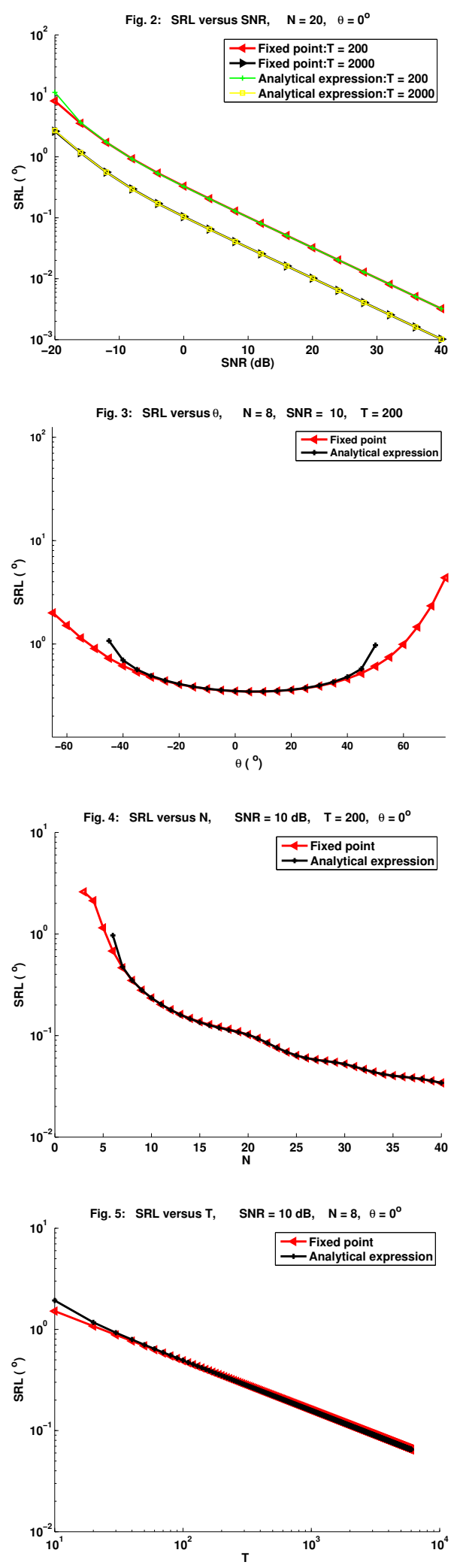


\section{REFERENCES}

[1] A. J. den Dekker, A. van den Bos, "Resolution, a survey," Journal of the Optical Society of America, vol. 14, pp. 547-557, 1997.

[2] S. T. Smith, "Statistical resolution limits and the complexified Cramér Rao Bound," IEEE Transactions on Signal Processing, vol. 53, pp. 1597-1609, 2005.

[3] R. Boyer, "Performance Bounds and Angular Resolution Limit for the Moving Colocated MIMO Radar," IEEE Transactions on Signal Processing, vol. 59, pp. 1539-1552, 2011.

[4] D. Swingler, "Frequency estimation for closely spaced sinsoids: Simple approximations to the Cramer-Rao lower bound," IEEE Transactions on Signal Processing, vol. 41, pp. 489-495, 1993.

[5] J.-P. Delmas, H. Abeida, "Statistical resolution limits of DOA for discrete sources," in Proceedings ICASSP, vol. 4, pp. 889-892, 2006.

[6] Z. Liu, A. Nehorai, "Statistical angular resolution limit for point sources," IEEE Transactions on Signal Processing, vol. 55, pp. 5521-5527, 2007.

[7] M.N. El Korso, R. Boyer, A. Renaux, S. Marcos, "Statistical analysis of achievable resolution limit in the near field source localization context," Elsevier Signal Processing, vol. 92, pp. 547-552, February 2012.

[8] M.N. El Korso, R. Boyer, A. Renaux, S. Marcos, "Statistical resolution limit of the uniform linear cocentered orthogonal loop and dipole array," IEEE Transactions on Signal Processing, vol. 59, pp. 425-431, 2011.

[9] A. Amar, A.Weiss, "Fundamental limitations on the resolution of deterministic signals," IEEE Transactions on Signal Processing, vol. 56, pp. 5309-5318, 2008.

[10] M. Shahram, P. Milanfar, "On the resolvability of sinusoids with nearly frequencies in the presence of noise," IEEE Transactions on Signal Processing, vol. 53, pp. 2579-2588, 2005.

[11] A. Khamsi Mohamed, A. Kirk William, An Introduction to Metric Spaces and Fixed Point Theory, Wiley, 2001.

[12] M.N. El Korso, R. Boyer, A. Renaux, S. Marcos, "Statistical resolution limit for multiple parameters of interest and for multiple signals," in Proceedings ICASSP, pp. 3602-3605, 2010.

[13] M.N. El Korso, R. Boyer, A. Renaux, S. Marcos, "Statistical resolution limit for the multidimensional harmonic retrieval model: hypothesis test and Cramr-Rao
Bound approaches," EURASIP Journal on Advances in Signal Processing, special issue on Advances in Angleof-Arrival and Multidimensional Signal Processing for Localization and Communications, pp. 1-14, Jun 2011.

[14] P. Stoica and R.L. Moses, Spectral Analysis of Signals, Prentice Hall, NJ, 2005.

[15] Cs. J. Hegedüs, "Laurent expansion of an inverse of a function matrix," Periodica Mathematica Hungarica, vol. 6, pp. 75-86, 1975.

[16] A.J. Weiss, B. Friedlander, "Range and bearing estimation using polynomial rooting," IEEE Journal of Oceanic Engineering, vol. 18, pp. 130-137, Apr. 1993. 